\title{
A CRITICAL REVIEW
}

\section{VITAMIN B DEFICIENCY AND NERVOUS DISEASE*}

\author{
BY
}

\section{D. ARING and T. D. SPIES}

AfFEctions of the nervous system which result from inadequate nutrition have assumed an increasingly important position through the rapid accumulation of clinical, experimental, and pathological data. The artificial synthesis of a number of the components of the vitamin B complex has made available pure crystalline material in large amounts for clinical research, and thus a milestone in the history of these affections has been passed. It now seems appropriate to review and summarize the knowledge of these important substances.

To evaluate the status of a nutritional deficiency in man is difficult. As a rule the patient induces his own disease and provides a deficiency that is not quantitatively measurable, but frequently clinical and laboratory evaluations are attempted. Both the laboratory method and the clinical method have certain advantages. Mice are not yet men and until they are the work coming from the study of human beings is essential. Since the experience of investigators working with animals may often point the way for clinical research, we have interspersed in this review a few of the pertinent studies on the effect of nutritional deficiency on the nervous system of animals.

A scheme for illustrating the multiple factors of the vitamin B complex is given in Fig. 1. To date the following portions of the vitamin B complex have been isolated and synthesized in amounts sufficient to allow for clinical trial : thiamin hydrochloride, nicotinic acid, riboflavin, and 2-methyl, 3-hydroxy, 4, 5-di (hydroxymethyl) pyridine. The deficiencies of these substances as they apply to the nervous system of man will be discussed briefly.

\section{Method of study}

Methods of control in the clinical study of the nutritional deficiencies are possibly the explanation for the varying results obtained in the treatment of these diseases from clinic to clinic and from physician to physician. Briefly our method has been to place under observation any patient suffering from nutritional deficiency immediately after admittance to the hospital or clinic. The history and physical examinations usually are sufficient to make the

* From the Department of Internal Medicine and Neurology, University of Cincinnati College of Medicine, and the Cincinnati General Hospital ; and the Nutrition Clinic of the Hillman Hospital, Birmingham, Alabama. 
diagnosis. Borderline cases or those of the subclinical variety are not selected for clinical study unless the patient is a remission from a previous attack. We have found that certain objective laboratory tests are useful and reliable indices of specific deficiency states. Since disturbance in porphyrin metabolism is

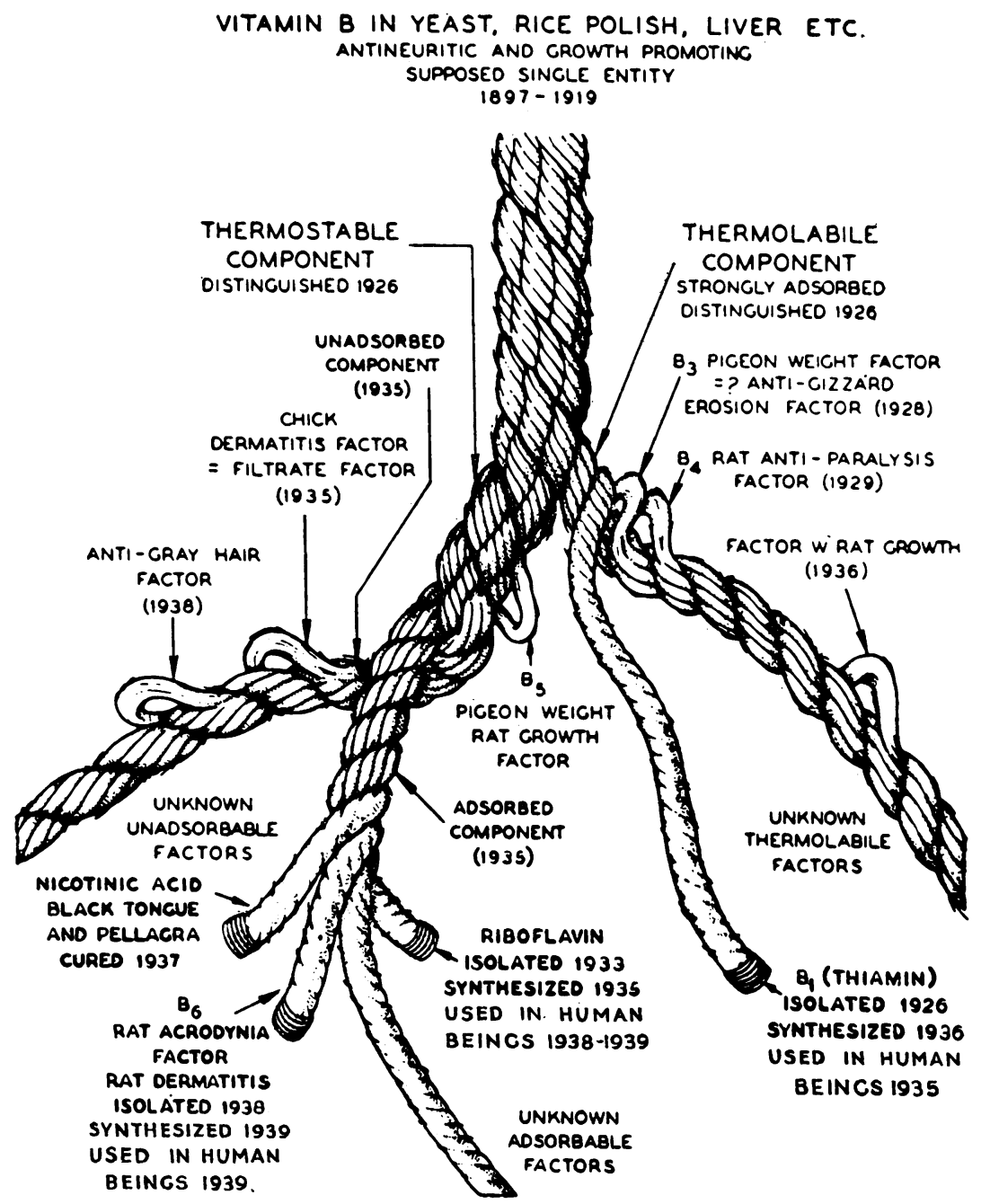

Fig. 1.-Differentiation of the components of Vitamin B complex. The components that have been isolated in a pure state are indicated in heavy lettering ; those which have been recognized only by the discrepancies in physiological properties of various crude extracts are indicated as loops ; the possible still unknown factors are divided into three main strands according to the principle of adsorbability which has been so useful in differentiation experiments.

often an important part of the deficiency syndromes, the urine of each patient is examined to determine the excretion of porphyrin or other ether-soluble red pigments which can be extracted in 25 per cent. $\mathrm{HCl}$. Using a bacteriological method, determinations are made of the concentration in the body fluids of 
cozymase, an enzyme fundamental to cellular respiration; the blood and urine of pellagrins contain only a fraction of the normal concentration of this enzyme. Studies are also made of the urinary excretion of vitamin B1, and a decreased output is suggestive of both beriberi and pellagra. The progress of symptoms over a period of days is noted while the patient is maintained, without therapy, on a restricted diet or on the identical diet that produced the deficiency. A rigid check is made of the dietary of all patients entering the Nutrition Clinic of the Hillman Hospital, Birmingham.

If no improvement in the symptoms of the nutritional deficiencies occurs under these conditions, they serve as an index for testing the potency of therapeutic agents. A specific therapeutic agent is of value if the symptoms of the deficiency clear promptly in all cases when sufficient amounts of the pure substance are added as a supplement to the control diet, all other conditions remaining constant.

It is probably superfluous to add that in the clinical study of the patients with nutritional deficiencies it is advisable to use only one therapeutic agent at a time. Another point to be considered is the fact that the patient may be improved by merely resting in bed.

To avoid the benefit of suggestion as much as possible, we substitute similarly appearing medicaments for the specific substance and observe the result.

\title{
Vitamin B1 (thiamin, aneurin, catorulin) deficiency
}

It is generally agreed that a prolonged deficiency of vitamin B1 in man may result in peripheral neuritis. By the term "neuritis" is meant disease of the neurone (Brown, 1938). The principal causes of neuritis may be grouped under six inclusive headings, as has been done by Cobb and Coggeshall (1934). As the ætiological agents causing neuritis are better understood, such clinical classifications will be replaced by classifications based solely on ætiology. Although this classification is arbitrary and imperfect as a result of the present state of our inadequate knowledge, it simplifies the understanding of the problem of neuritis. The types of neuritis as noted by Cobb and Coggeshall will be considered, and information and experience in the therapy of these diseases with vitamin Bl will be noted. In the table, peripheral neuritis has been divided into local and general, and in this manner the unilateral disorders affecting one nerve are segregated from the large group of systemic disorders which cause multiple neuritis.

\section{Localized Neuritis}

Mechanical
Pressure
tumour
oedema
arthritis
fibrosis
Trauma
Saturday-night paralysis
Volkman contracture
Meralgia paresthetica

\author{
Infections \\ Diphtheria \\ Tetanus \\ Streptococci \\ Leprosy
}

Volkman contracture

Meralgia paresthetica 
Localized neuritis usually occurs in ambulatory patients, and mechanical or infectious ætiological agents can be determined by clinical methods. The symptoms usually respond to medical and surgical therapy. Relief of localized neuritis following the administration of crystalline vitamin B1 has been reported by some investigators. Such reports, however, tend to be misleading, since physicians who obtain negative results are not prone to report them. Furthermore, the number of cases which have been reported is small and, in general, the studies are not adequately controlled. Hence the results need confirmation. Our unfinished studies show that some cases are relieved by thiamin and others are not. No conclusive reports have as yet appeared in the literature establishing the value of vitamin B1 in the prevention of localized neuritis.

We have observed the relief of the acute pain following the use of vitamin B1 in a case of brachial neuritis which developed six weeks after mastectomy. This case report is given because the results of the vitamin therapy of apparently similar cases are so confusing and the experiences of various men contradictory.

A female, age 44, on 13th September, 1938, had a simple mastectomy under cyclopropane anæsthesia, for chronic cystic mastitis, at the Cincinnati General Hospital. Twelve hours after the operation there was pain in the arm on the same side ; this arm had been strapped to the side of the patient during the operation. The extremity felt heavy until six weeks later, when the patient awoke one morning with severe pain in the arm (after taking a long automobile trip during which she had done no driving). There was numbness of the first and second fingers and weakness of the entire extremity. All symptoms were aggravated by use of the extremity, and were worse at night.

The patient was examined by one of us on 4th December, 1938, $2 \frac{1}{2}$ months after the operation. The pain which had been present constantly was not relieved by analgesics. There was no history of obvious dietary deficiency, although she had lost her appetite and had vomited occasionally after the onset of the acute pain. She had lost $15 \mathrm{lb}$. in weight since the operation.

Neurological examination revealed atrophy of the musculature of the forearm. All movements of the affected extremity were reduced in speed and volume. She complained of continuous numbness and tingling of the hand and fingers. Manipulation of any of the muscles of the extremity caused pain. Pressure in the axilla or in the supra-clavicular space caused great pain. The trapezius muscle on the same side was tender. The only demonstrable loss of sensation was that to light touch in the skin of the distal joints of the fingers and thumb on the affected side. The tendon reflexes were normal.

An injection of $50 \mathrm{mg}$. of thiamin hydrochloride was given intravenously in sterile physiological solution of sodium chloride daily for three days. She slept well on the first night after the medication, whereas previously she had been unable to rest despite large doses of codeine, emperin, nembutal, bellergal, or sodium phenobarbital. All sedatives and analgesics were discontinued after the third injection, and the patient was then allowed to go three days without thiamin. On the third day, the constant aching pain had returned, but again was relieved by the intravenous injection of $50 \mathrm{mg}$. of thiamin hydrochloride. This dosage was repeated daily until a total of $350 \mathrm{mg}$. had been given. The movements of the extremity became stronger, although not as strong as those of the unaffected extremity. She had occasional mild pain as long as 5 months after the onset, but it was not severe enough to require analgesics or intravenous vitamin B1 after the seventh injection. She continued to improve, and 8 months after the onset had practically recovered. 
The criteria at our command led us to classify this patient as a case of localized mechanical neuritis. The result obtained with thiamin was prompt and satisfactory. In fact, the patient was uncomfortable with medicaments other than thiamin. Cases such as this illustrate our incomplete knowledge of this subject, and a reasonable explanation of their physiological pathology cannot be given with the data at hand.

\section{Generalized Polyneuritis}

Measles
Small pox
Chickenpox
Parotitis
Herpes
Acute febrile (infectious
$\quad$ or infective)

Virus

$$
\begin{aligned}
& \text { "Landry's" } \\
& \text { Poliomyelitis } \\
& \text { Encephalomyelitis } \\
& \text { Epidemic (lethargic) } \\
& \text { encephalitis } \\
& \text { Erythro-edema } \\
& \text { Acute rabic myelitis }
\end{aligned}
$$

The virus diseases listed above are often characterized or accompanied by peripheral neuritis as a late complication. No crucial observations have been made to determine whether faulty nutrition plays a role in the development of the neuritis seen in these diseases. The evidence available tends to show that vitamin B1 does not play any direct role in counteracting the effects of these infectious agents.

Our experience with three cases of infectious polyneuritis has been that they have had progression of some of their neurological signs despite large doses (50 mg. twice a day) of thiamin intravenously. In two of these cases this therapy was continued for 1 month.

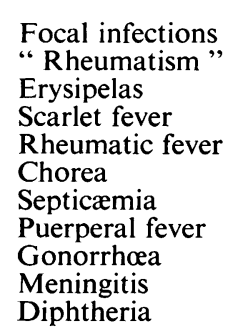

\section{Bacteriotoxic}

Typhoid fever
Paratyphoid fever
Typhus fever
Influenza
Pneumonia
Malaria
Relapsing fever
Serum sickness
Acute enteric fever
Syphilis

The diseases in this group often interfere with proper nutrition and thus predispose the patient to development of a deficiency of vitamins. Many patients with infection also have an increased requirement for certain essential nutritive substances. In typhoid fever, for example, the requirement for vitamin B1 is raised because of the high fever, loss of appetite, or excessive diarrhœa. The incidence of peripheral neuritis in patients with typhoid fever was high a few decades ago, when an effort was made to restrict the diets of such patients. The great decrease in the incidence of peripheral neuritis in typhoid fever in recent years is probably due to the prescription of adequate diets in the treatment of that disease. In fact, it is becoming uncommon to see neuritis incident to the bacteriotoxic diseases, probably because of the increasing awareness of the importance of proper nutrition in the ill. We have little 
information concerning the role of vitamin B1 deficiency in the development of any of the neuritides accompanying bacteriotoxic diseases, but a number of reports have appeared indicating that the administration of vitamin B1 often relieves the symptoms of neuritis in these cases. The neuritis of acrodynia possibly belongs under this heading. Durand, Spickard, and Burgess (1939) reported the cure of acrodynia in two children following the intramuscular injections of vitamin B1.

Mercury
Lead
Silver
Arsenic
Phosphorus
Methyl alcohol
Ethyl alcohol
Ethyl iodide
Trichlorethylene
Carbon tetrachloride
Trinitrotoluene
Dinitrobenzene

\section{Chemical}

Triorthocresyl phosphate

Aniline

Sulphonethylmethane barbital, etc.

Chloral, chlorbutanol

Carbon monoxide

Carbon bisulphide

Thallium

Sulphur

Emetin

Gold

Bismuth

Sulfanilamide

The course of two cases of triorthocresyl phosphate poisoning (Jamaica ginger paralysis) two weeks after the onset of symptoms was unaltered by the use of large amounts of crystalline vitamin $\mathrm{B} 1 *$ by mouth. One of the patients received $20 \mathrm{mg}$. daily for a period of 1 month and the other received $500 \mathrm{mg}$. daily for the same length of time. The symptoms of neuritis progressed despite this therapy, and signs of involvement of the pyramidal tracts became obvious within 3 weeks after the onset of the neuritis. It is theoretically possible that large amounts of vitamin $\mathrm{B} 1$ crystals given before the ingestion of triorthocresyl phosphate might act as a protective agent. It has been reported that the administration of vitamin B1 causes relief of the symptoms of the neuritis following exposure to toxic agents such as nicotine, lead, thallium, arsenic, and mercury. As yet, however, there is no conclusive evidence that the lack of vitamin B1 plays a major role in the development of any of the "chemicai neuritides."

A case report is appended to illustrate the improvement that may be obtained in neuritis considered to be of chemical origin.

A man of 40 had experienced the onset of diarrhœa in mid-May of 1938, which was continuous through August 1938. Despite the daily occurrence of diarrhœa, which was accompanied by some cramping of the abdomen, the patient remained at hard work every day during this period. Repeated stool examinations were negative, but despite this a thorough course of emetine was begun in August as the patient was suspected of having amœbic dysentery. It is thought that about $\frac{3}{4}$ grain was taken by injection at a single dose and there had been 10 doses. The diarrhœa ceased after the first dose of emetine. In early September he was able to get away from work for two weeks. On returning from this holiday, he observed the occurrence of a peculiar stinging in the inner surface of the sole of the left foot. This paresthesia ascended the foot in a rather indefinite manner, following no nerve or segment distribution. Shortly after the occurrence of this numbness and paresthesia in the left foot, he observed the same thing in the right foot and the peculiar sensation ascended the left leg and finally

\footnotetext{
* Furnished through the courtesy of Merck and Company.
} 
involved all of both legs and a portion of the lower thigh. There had also been some numbness about the left hip. There had been no difficulty whatsoever with the upper extremities or about the head and face. He described the sensation as diminished sensation in the lower extremities (feeling as though the feet had been asleep and were just coming out of this state). He stated that there was no complete loss of sensation anywhere. Underneath this sensory loss there was a dull aching which went unnoticed if he concentrated on some other subject. The lower extremities felt better if he shifted their position rather frequently when sitting. If he walked as far as 400 yards he noticed that the aching became more obvious and his legs tired. There had been no bladder disturbance. There was no stiffness in the extremities. He believed that the motor power was good and near normal in the lower extremities. His gait was no different than before the onset of difficulty in his lower extremities.

It is important to note that the food intake had been considered to be good throughout the illness, that is, since May and before, and that the beginning of his neurological symptoms occurred just about two weeks after the last injection of emetine.

Neurological examination was performed on 25th October, 1938.

Cranial Nerves.-Movements of the eyes were full in all directions; there was no nystagmus. Sensation of the face was normal throughout. Movements of the jaws and face were normal. Movements of the neck were strong to either side. The tongue protruded in the midline.

Motor System.-There was no loss of motor power in any of the extremities. Tone was normal and muscle development was excellent throughout. The gait was essentially normal. He walked on a rather broad base and his toes pointed outward, but he walked well, raising his feet properly. He did not have to watch his feet.

Sensory System.-There was slight tenderness to deep pressure over the nerve trunks and in the muscles of the legs, especially the calf muscles. Sensation in the upper extremities, including the appreciation of vibration, was normal with the exception of slight diminution of pain and touch in the distal two joints of all of the fingers. In the lower extremities there was loss of sensation in the lumbar segments below the mid-thigh. This was not a complete loss, and sensation improved in an ascending manner. The appreciation of temperature, pain, and touch was similarly affected. There was hyperæsthesia of the first and second sacral segments in the lower extremities. The appreciation of vibration was almost completely absent in the lower extremities below and including the patellas. The appreciation of passive changes in position of the toes was very good. Tendon reflexes in all of the extremities were normally active. There was no clonus. An occasional response on testing for a right Gordon was obtained which was as follows : the right great toe dorsi-flexed slightly without any fanning of the other toes. The other plantar responses were "flexor" in type.

The excellent and detailed history which this patient gave suggested that the cause of the neuritis was emetine rather than nutritional deficiency. The patient had an excellent appetite throughout his illness. His food intake was exceptionally good, and the diarrhœa had remitted two weeks before the onset of the neuritis, when he was on a holiday and apparently in good health.

Treatment with vitamin B1 was begun five weeks after the onset of the neuritis ; $100 \mathrm{mg}$. of thiamin was given intravenously twice daily. Beginning of relief occurred within 12 hours after the first injection. This dosage was maintained for 3 days with great relief of the paresthesias, aching, and fatigue. Thiamin was then administered by mouth in amounts of from 100 to $500 \mathrm{mg}$. daily for three weeks. For two days at the end of this period, when the patient was taking these large amounts of thiamin by mouth, he was given a supplementary injection of $100 \mathrm{mg}$. intravenously twice per day. He noted that he 
received additional benefit from the intravenous injection and that the oral administration of vitamin B1 could not compare in its effect to that obtained from the intravenous administration. Thiamin was continued by mouth until the patient had had three months of treatment, at the end of which time he was completely relieved of symptoms.

We have been unsuccessful in treating the neuritis in the upper extremities of a young man, a worker with refrigerants. Repeated large intravenous injections of vitamin B1 had no effect on the course of the neuritis, which was probably due to methyl chloride.

\begin{tabular}{ll}
\multicolumn{1}{c}{ Nutritional Deficiency or } & Metabolism \\
Pellagra & Cancer with cachexia \\
Pernicious anemia & Tuberculosis with cachexia \\
Sprue & Senility with cachexia \\
Beriberi & Diabetes \\
"Alcoholic neuritis" & Myxedema \\
"Korsakoff's psychosis" & Hematoporphyrinuria \\
Pernicious vomiting & "Recurrent polyneuritis " \\
Hunger œdema & "Chronic progressive \\
Pregnancy & polyneuritis" \\
Chronic colitis & Chronic bacillary dysentery
\end{tabular}

There is a long list of diseases in which polyneuritis, when it occurs, is directly related to the lack of proper nutrition. This list is composed chiefly of metabolic and deficiency diseases which often have a number of clinical features in common. In the scope of this article we are concerned chiefly with the neuritic manifestations which may affect patients having any of these diseases.

Evidence is accumulating which indicates that many of the neuritic symptoms listed under various syndromes are clinical variations of one large ætiological group. Since vitamin B1 will prevent and usually relieve the manifestations of the polyneuritis of beriberi, a known dietary deficiency disease, and since it is essential for the integrity of the nervous tissue in general, it seems likely that its absence may play a very important part in the development of neuritis in a number of these diseases in addition to beriberi. Evidence indicates that vitamin B1 is a useful therapeutic agent in the treatment of "alcoholic" neuritis, with or without pellagra, polyneuritis of pregnancy, and the neuritis of diabetes. Most of the other diseases under this heading are related to disturbances of nutrition, and it may well be that a lack of vitamin B1 plays a role in the production of the neuritic manifestations which so frequently accompany them. These diseases of metabolism are often associated with loss of appetite and malabsorption, conditions which, in themselves, may lead to a deficiency of some specific factor or factors.

The clinical manifestations of these diseases are extremely variable. Studies of a large group of people who are addicted to alcohol and who are also malnourished show that some individuals have no symptoms; that some have symptoms of beriberi ; and that others have nervous, dermal, and gastrointestinal changes characteristic of pellagra. Still others will show hæmatological and neurological changes similar to those associated with sprue and pernicious anæmia. Some of the persons addicted to alcohol develop Korsakoff's syndrome. This clinical syndrome is characterized by a peripheral 
neuritis which is identical with that of beriberi and by a psychosis often resembling the mental changes seen in pellagra.

Pellagra.-The pellagra syndrome conspiciously affects the alimentary tract and the dermal and nervous systems. The symptoms arising from involvement of the nervous system, like the other symptoms of pellagra, do not appear in any regular order. They arise from cerebral involvement, degeneration of the spinal cord, and peripheral neuritis. The mental changes may be clinically identical with those of Korsakoff's psychosis, or in fact the symptoms may manifest themselves as any of the well-known psychotic types. The peripheral neuritis is clinically indistinguishable from the polyneuritis of beriberi and chronic alcoholism.

In the Nutrition Clinic at the Hillman Hospital, Birmingham, Alabama, there are under observation 700 pellagrins. In these cases it has become increasingly evident that the inadequate and unbalanced diet eaten by these patients predispose them simultaneously to a number of nutritional disorders. Thus we have found that pellagrins almost without exception suffer from an associated beriberi at some time during the course of their disease. The neuritis from which the ambulatory pellagrin suffers is not severe and it is usually limited to the lower extremities.

Spies and Aring (1938) studied the perıpheral neuritis of pellagra and demonstrated that it could be relieved with thiamin, while other symptoms of pellagra remained stationary or progressed. These observations have been repeated on numerous occasions. The pain and numbness associated with the peripheral neuritis can always be relieved by the use of crystalline vitamin B1. The patients attending the nutrition clinic subsist on the same diet as they had been taking before coming under observation. Despite this fact, the symptoms of neuritis in the ambulatory pellagrin subside rapidly following the injection of $50 \mathrm{mg}$. of vitamin B1 on two successive days.

Pernicious AnÆmia.-About 75 per cent. of all cases of Addisonian pernicious anæmia have involvement of the nervous system, and in at least 25 per cent. of all cases neural or mental symptoms are the first indications of the disease. The symptoms arising from involvement of the nervous system are usually characterized by disorders of sensation, especially tingling of the hands and feet. About 10 per cent. of all patients having symptoms arising from the nervous system have degeneration of the posterior and lateral columns of the spinal cord. The peripheral neuritis in patients with pernicious anæmia cannot be distinguished pathologically (Greenfield and Carmichael, 1935) from the peripheral neuritis associated with pellagra, chroric alcoholism, and other conditions described above. It has been shown that the progression of the spinal cord and peripheral nerve lesions in pernicious anæmia can be averted by adequate amounts of a crude liver extract administered intramuscularly. Liver extracts administered a few years ago were considerably less refined than some that are now being dispensed and it may be that the more refined concentrates now in use will not protect against neural involvement. This point should be determined within the next few years. In general, it is believed that the more crude the liver extract, the greater is the content of vitamin B1. 
Schiro, Spies, and Aring (1939) studied three cases of pernicious anæmia with signs of spinal cord degeneration and peripheral neuritis. With the administration of large amounts of brewer's yeast by mouth a reticulocyte response was obtained in these cases, and the red blood cells increased in number. Meanwhile the neurological signs progressed relentlessly and became so severe that the study had to be terminated. This was done by the use of large doses of liver extract, which halted the progress of the neurological signs. Before terminating the study in two cases, thiamin was given intravenously in 50 -mg. doses twice per day for 10 days as a supplement to the yeast. This therapy had no effect on the progression of neurological signs over this short period. The peripheral neuritis in these cases of pernicious anæmia was complicated by the spinal cord lesions, which made evaluation difficult and limited the period over which therapy other than liver extract could be tried with safety.

At the present time the physician will do well to use the crude liver extracts, which have been proved to be effective in the treatment of spinal cord lesions associated with pernicious anæmia. Vitamin B1 preparations are, of course, theoretically valuable accessories and their use should not be denied.

DiABETES.-Attempts to explain the high incidence of neurological lesions in diabetes began with the original concept, suggested by Bernard's work, that the nervous lesions were the cause rather than the result of disturbed sugar metabolism. By the end of the first decade of the insulin era, arterio-sclerosis and vitamin deficiency were believed by most writers to rank high among the ætiological agents responsible for the neurological lesions.

Since the polyneuritis of diabetes and of beriberi may give rise to similar or identical symptoms, it was natural that crystalline vitamin $\mathrm{B} 1$ should be given to patients with diabetic neuritis. Published reports to date are surprisingly few, and though suggestive they are far from conclusive. Vorhaus, Williams, and Waterman (1935) included diabetic neuritis in a group of metabolic neuritides which improved following crystalline vitamin B1 therapy. Sciclounoff and Broccard (1936) described excellent results in five out of six cases of diabetic neuritis treated with preparations of crystalline vitamin B1 administered parenterally. Their case reports said little about concurrent diabetic control. One can feel confident that vitamin B1 deficiency does occur in certain diabetics, but what percentage of the cases of diabetic neuritis have vitamin B1 deficiency cannot be estimated with any degree of assurance. Nor can we state whether or not local ischæmia resulting from sclerosis of the arteries might predispose to some of the manifestations.

At the present time it seems that the prophylaxis against neuritis in diabetic patients, in addition to painstaking control of the diabetes, requires the prescription of diets adequate in the antineuritic vitamin. Sindoni (1936) has shown that several typical diets for diabetic patients are actually deficient in this vitamin. The diets used in the clinical management of diabetics in many hospitals are on the borderline of normal requirements and would be inadequate in the event of any additional requirement.

Since diabetes and vitamin B1 deficiency are common, the two will some- 
times occur by coincidence in the same person. At the present time information is inadequate to state that the majority of cases of diabetic neuritis are caused by an inadequate amount of vitamin B1. The following theoretical considerations, however, favour the hypothesis that vitamin B1 deficiency may play a part in the ætiology of these changes : (1) the close relationship between vitamin B1 and carbohydrate metabolism ; (2) the similarity of the clinical and neuropathological pictures between diabetic neuritis and the neuritis associated with other diseases which are thought to be due to a lack of vitamin B1; (3) the realization that many diabetics subsist on diets which are low in vitamins.

TUBERCULOSIS.-Some investigators have suggested that the neuritis accompanying tuberculosis is associated with the accompanying emaciation. It is not uncommon to find neuritis associated with late tuberculosis, especially in institutions. We observed that the clinical manifestations of neuritis in three persons with tuberculosis were identical with those of the peripheral neuritis associated with the other diseases described here. The neuritis in these three patients developed while they were in the hospital and gradually disappeared following the administration of large amounts of yeast and a wellbalanced diet.

PoRPHYRINURIA.- It has been observed for some time that increased quantities of porphyrin may be excreted in the urine in febrile conditions, liver diseases, anæmia, and in poisoning by sulphonal, veronal, trional, lysol, chloroform, salvarsan, zinc, acetanilid, tin, and lead. Some writers regard this finding as a simple increase in the amount of normal porphyrin excretion resulting from the underlying disturbances in metabolism. In recent years it has been shown (Beckh, Ellinger, Spies, 1937) that a number of the metabolic and deficiency diseases which are accompanied by peripheral neuritis also are associated with the porphyrinuria and other abnormal urinary pigments. It seems likely that porphyrinuria occurs as a physiological process and that the amount excreted may be increased in certain diseases and intoxications. Very rarely porphyrinuria may be the chief symptom of a characteristic clinical syndrome of unknown ætiology. When this disease affects the nervous system, the first symptom is severe and constant pain in the extremities. This is usually accompanied by muscular weakness or flaccid paralysis. Pathological examination shows widespread degeneration of the myelin sheaths in the peripheral nerves and spinal cord. At present there is little information as to whether vitamin B1 bears a relationship to any of the neuritides associated with porphyrinuria or with the other abnormal pigments which are ethersoluble and give red colour in 25 per cent $\mathrm{HCl}$. These urinary pigments do seem to accompany malnutrition.

Sporadic Cases of Beriberi in Association with Malnutrition.Sporadic cases of beriberi are reported in the literature from time to time. However, isolated cases are often not recognized as true beriberi. Kepler (1925) reported a case in a negress who restricted herself to a diet of raw starch. At the end of three years she developed polyneuritis with œdema and an enlarged heart. We have observed the development of beriberi in 15 cases who restricted their diet to carbohydrates and fats. Following treatment with large 
amounts of yeast, the symptoms disappeared. Urmy, Ragle, Allen, and Jones (1934) demonstrated that beriberi may follow a short-circuit operation on the small intestine and may be cured by disconnecting the intestinal anastomosis. Their cases show that beriberi may result from a lack of proper absorption. Many persons suffering from malnutrition have signs and symptoms clinically indistinguishable from those of beriberi.

Since most physicians rarely consider beriberi in differential diagnoses, we contend that the disease occurs much more commonly than is generally thought and, in fact, that it is endemic rather than sporadic.

The diagnosis of beriberi may be made if the patient has evidence of peripheral neuritis, with or without œdema, cardiovascular or gastrointestinal abnormalities, and a history of a diet deficient in vitamin B1. The laboratory is of little aid in the diagnosis of thiamin deficiency, as there is as yet no simple chemical test for the vitamin.

Williams and Spies (1938) have described in review the important work which led to the concept that certain types of neuritis are due to nutritional deficiency. The identification and synthesis of vitamin B1 by Williams and his associates $(1934,1936)$ made available large quantities of crystalline vitamin B1 and led to rapid progress in clinical research on this subject.

We have had experience with the neuritis of pellagra, pernicious anæmia, beriberi ("alcoholic and non-alcoholic neuritis"), tuberculosis, and pregnancy. These neuritides, excepting possibly the neuritis encountered in pernicious anæmia, respond promptly to large doses of vitamin B1. We usually administer thiamin intravenously, giving $50 \mathrm{mg}$. twice per day for 1 week. The initial response to this therapy is usually rapid. Pain is greatly relieved within 24 hours, and the strength, accuracy, and the speed of movement is usually improved within 3 days, this improvement possibly being due to the conspicuous relief of pain. If analgesics have been used, they may be discontinued at this time and patients who have slept little or not at all for weeks may be able to sleep with no medication, other than vitamin B1. The relief which usually occurs in this group of nutritional deficiency neuritides is abrupt and dramatic. Patients previously bedfast may be able to walk within a few weeks. However, the patient quickly reaches a stage beyond which thiamin will not cause further improvement, and residual changes may persist indefinitely.

Nothing more than the relief of pain and slight early improvement in motion may be expected from vitamin B1 therapy in the extremely severe case of neuritis in which there has occurred practically complete paralysis of all of the extremities. These patients run their natural course of recovery, which requires months and even years, with or without vitamin therapy.

It is our thought that the initial prompt improvement with thiamin in cases of nutritional deficiency may be humoral in nature. This impression is furthered by the work of Minz (1938), who has been able to show that vitamin B1 enhanced the effect of acetylcholine in the transmission of the nervous impulse. It seems that vitamin B1 inhibits cholinesterase in the same manner as does eserine. In our patients in which tests for cholinesterase were performed (in 
vivo), the serum cholinesterase tended to be inhibited by the administration of thiamin. Glick and Antopol (1939) obtained similar results with horse and rat serum (in vitro), and they made the important observation that the inhibition of the serum cholinesterase was obtained only with concentrations of vitamin B1 in excess of those known to occur in the living organism.

We have studied the peripheral nerve histologically by the biopsy method in 12 cases of deficiency disease with neuritic symptoms, in several instances both before and months after treatment with crystalline vitamin B1. In all cases marked loss and degeneration of myelin sheaths was observed in the terminal portions of the internal branch of the anterior tibial nerve. The axones in these peripheral nerves have been, on the whole, normal. In those cases in which the peripheral nerve was examined as long as 11 months after treatment was instituted, the loss of myelin sheaths was severe.

This study lends support to the hypothesis that the initial spectacular improvement following vitamin B1 therapy, which is experienced by sufferers from nutritional neuritis, is on a humoral basis. It also illustrates how slowly restitution of the nerve may occur.

\section{Use of Cocarboxylase}

In 11 cases of moderate nutritional neuritis the administration of cocarboxylase * (phosphorylated thiamin), $10 \mathrm{mg}$. in sterile physiological solution of sodium chloride twice daily, by intravenous injection, resulted in the same improvement that had been seen following the use of thiamin. This result supports the hypothesis that vitamin B1 may be utilized as cocarboxylase by the body (Spies, 1939). Cocarboxylase is a fundamental oxidative-reductive enzyme in the body. Lewy, Himwich, Frostig and Spies (1939) have described that " the administration of 50 milligrammes of cocarboxylase (Merck) to persons who exhibited signs of an 'active process' was followed, in every instance, by dramatic and rapid improvement. The bisulfite-binding substances decreased in quantity. The pathological signs of peripheral and cranial nerves, which had become increased in patients who were being treated with nicotinic acid and riboflavin, became less conspicuous or disappeared. The irritability of a number of muscles, measured in terms of their strength-duration curves, returned from underexcitability before treatment to normal values and in some cases even progressed to overexcitability. Correspondingly, the increased threshold of sensibility to touch and prick in arms and legs was reduced to normal, and the depressed pupillary and corneal reflexes improved quantitatively, often becoming normal.

Following the administration of cocarboxylase, some beneficial effect occurred, in some persons within an hour and in all persons within four hours. Improvement continued for from one to four days thereafter. Since the unbalanced diets of these persons remained essentially unchanged, in the absence of further therapy the patients tended to regress rapidly to their condition preceding treatment. A psychoneurotic syndrome which was recognized in these persons and which responded promptly to the administration of cocarboxylase

\footnotetext{
* Furnished through the courtesy of Merck and Company.
} 
will be described separately. In contrast, in the persons selected for control, there was no decrease of bisulfite-binding substances in the book and no improvement in the neurological and psychoneurotic symptoms following cocarboxylase therapy."

\section{Wernicke's Pseudo-encephalitis}

Wernicke's pseudo-encephalitis has been produced by Alexander, Myerson, and Pijoan (1938) in the pigeon by the feeding of diets low in vitamin B1 and high in other vitamins (A, B, C, and D). They have not produced the disease by a diet low in vitamin $\mathrm{Bl}$ except in rare instances. The feeding of an excess of the other vitamins seems to be a requisite in the production of Wernicke's disease in their experience. Wernicke's disease is rarely encountered in man, and when its clinical incidence is reported in large numbers the diagnosis should be accepted with caution. The work of Alexander, Myerson, and Pijoan warrants a trial of thiamin in these cases, though these workers have not yet attempted to cure their pigeons with vitamin B1.

\section{Use of Vitamin B1 in the Treatment of Pain and of Tabes Dorsalis}

Vitamin B1 probably has been administered to patients suffering from almost every kind of nervous disease. Like other new forms of therapy (insulin, liver extract, sulfanilamide), it has been applied in the attempt to treat many diseases.

Our experience with patients suffering from severe pain (carcinoma, cord tumour, brachial and trigeminal neuralgia) was that thiamin, administered intravenously in 50-100 mg. daily over a period of 10 days, did not act as an analgesic. We have administered thiamin intravenously $(100 \mathrm{mg}$. in normal physiological saline solution) during the crises of tabes dorsalis in six patients. This procedure was recommended by Metildi (1939). We have observed complete relief of the crises for varying periods after the injection. In every case with the return of pain, normal physiological saline solution was given intravenously. The saline solution alone was as efficacious in the relief of tabetic crises as was the thiamin dissolved in saline solution. We have, as yet, no explanation for this phenomenon.

We are unable to evaluate the recommendation in the literature that other neurological conditions may be improved with the use of vitamin B1, but it is our belief that vitamin B1 is useful in the amelioration of these disorders only if they are caused by a deficiency of vitamin B1. It is often difficult to make a clinical diagnosis of vitamin B1 deficiency with any degree of assurance, but this defect may be remedied shortly by the development of simple diagnostic laboratory methods.

\section{Chemistry of Vitamin B1}

Thiamin hydrochloride in its phosphorylated form (cocarboxylase) is an important enzyme which plays a prominent role in oxidation and reduction. There is much to suggest that thiamin may act other than as a pyrophosphate. It seems that so far as the formation of the pyrophosphate is concerned the 
alcohol group of the thiazole side-chain is necessary. At the present time it is wise to suppose that thiamin is a unique or almost unique molecule which cannot undergo much alteration without impairment of physiological activity.

\section{Prevention and Treatment of Vitamin B1 Deficiency}

Vitamin B deficiency can be greatly decreased by the application of the following recommendations : (1) fresh foods such as potatoes, native vegetables, pork and liver, eggs, milk and fruits, and especially beans and whole grain cereals and dried or canned foods should be included in the diet whenever possible. (2) The use of dried yeast, wheat germ, liver, kidney, and vitamin B1 crystals as supplements to the diet should be encouraged whenever possible. The expense of such materials, however, often interferes with their extensive use. (3) Since vitamin B1 is water-soluble, a large amount of it is lost when the water in which the foods are cooked is thrown away. It is recommended, therefore, that the water in which foods containing vitamin B1 are cooked be used, for broths. Whole barley or other grains which are rich in vitamin B1 may be added to such broths to insure additional protection. (4) The use of undermilled rather than overmilled grains should be rigidly enforced among govern. ment troops and in prisons, asylums, and other institutions where mass feeding is required. Only about 6 per cent. of the vitamin B1 content remains in white flour. Each lot of grain purchased should be examined to determine whether there is an adequate amount of the germ and of the external layers of the grain. Since molds, weevils, and other infestations deprive cereals of this vitamin, one must be certain that the grain has not spoiled. (5) Particular attention should be directed toward the diets of persons with chronic debilitating diseases and increased metabolism, for the incidence of vitamin Bl deficiency is high among such people. Chronic alcoholic addiction, pellagra, sprue, pernicious anæmia, colitis, diabetes mellitus, tuberculosis, senility, malignancy, cirrhosis, and other diseases may interfere with the proper nutrition of the patient and thus predispose to the development of vitamin B1 and nicotinic acid deficiency. (6) The diets of pregnant and lactating women should be especially rich in vitamin B1. Whenever there is any doubt as to the adequacy or utlization of this vitamin either in the mother or in the child, supplements should be given. The supplements should be continued for the mother and child until the proper diet is assured. The nursing mother should receive at least $5 \mathrm{mg}$. of thiamin or its equivalent daily ; the infant should receive $0.5 \mathrm{mg}$. of thiamin or its equivalent, and children should receive $1 \mathrm{mg}$. or its equivalent per day. (7) The requirement for vitamin Bl may be distinctly above the average in persons with fever, severe gastro-intestinal symptoms, hyperthyroidism, and other conditions. It is essential for the physician to prescribe amounts above the average for such persons. The authors suggest for such a maintenance dose, from 5 to $10 \mathrm{mg}$. of pure vitamin B1 or its equivalent, except when the patient is unable to absorb from the gastro-intestinal tract. In such instances it is essential that vitamin B1 be given parenterally in order to protect the person from a deficiency of this vitamin. 


\section{Clinical Indications for Vitamin B1 Therapy}

The Council of Pharmacy of the American Medical Association (1936) has recently stated that it considers the following to be allowable claims for the use of vitamin B1 in therapy : (1) May be cited as of value in correcting and preventing absence or loss of appetite-anorexia-of dietary origin in certain cases. (2) Of value in securing optimal growth of infants and children. (3) Of value in correcting and preventing beriberi. (4) Because it is a dietary essential and its administration in concentrated form is of value in some conditions where difficulty in utilizing ordinary foods in the usual way is encountered. (5) There are many experimental indications in the literature indicating other possible functions of vitamin B1, e.g. an influence on intestinal motility and neuritis of various types and also indications of greatly augmented requirement when metabolism has increased as in hyperthyroidism, neuritis of various types, and infection. It seems too early to permit advertising claims for these items.

The authors support these claims and the method in which they are presented. However, we feel that since the time of their publication in 1936, additional information has become available which indicates that persons having polyneuritis associated with chronic alcoholism, pellagra, or pregnancy are greatly benefited by the administration of this material. There is enough clinical information available at the present time to warrant the trial of thiamin in patients who have peripheral neuritis secondary to dietary deficiency or associated with under-nutrition from any cause. Also a trial is warranted in peripheral neuritis and œdema of undetermined origin, though it is obviously too early to permit advertising claims for this latter group.

It is probable that in the near future, simple diagnostic clinical and laboratory methods for the determination of vitamin B1 deficiency will become available. Using a fluorescence method, Cline and his associates (1938) devised a method for determining excretion of vitamin $\mathrm{B} 1$ in the urine.

\section{Specific Treatment}

The essence of treatment lies in the administration of adequate amounts of foods rich in vitamin B1, supplemented if possible with large amounts of a specific therapeutic agent. The foods included in the dietary will depend upon the age, race, habits, tastes, and financial status of the patient concerned.

For an adult a well-rounded diet of 4,500 calories, including fresh native vegetables and fruits, whole-grain cereals, peas, beans, barley, pork and liver, eggs, milk, and other foods of high vitamin B1 value should be the objective. Whenever possible this diet should be supplemented with one or several of the following curative therapeutic substances, for even in chronic resistant cases which are cured slowly these substances are of benefit in relieving symptoms and in shortening convalescence : dried brewers' yeast (6 ounces daily); wheat germ (6 ounces daily); extract of rice polishings (tikitiki) (3 ounces daily); or crystalline vitamin B1 (10 mg. twice daily). In cases of severe beriberi even larger doses of crystalline vitamin B1 may be indicated. In such cases it seems wise to administer $20 \mathrm{mg}$. until the signs of avitaminosis have disappeared. In cases of mild deficiency doses of $5 \mathrm{mg}$. daily are adequate. 
Our experience leads us to believe that if crystalline vitamin B1 is to be effective in any very serious nervous disease it must be given in large doses. We recommend the administration of at least 50 to $100 \mathrm{mg}$. each day, given parenterally in several doses of 10 to $20 \mathrm{mg}$. each. It is our belief that if no response is obtained within a week, further vitamin therapy will be unavailing. There is practically no danger of overdosage with thiamin. We have administered $500 \mathrm{mg}$. intravenously daily for $1 \mathrm{month}$, and have given $500 \mathrm{mg}$. daily by mouth over a period of 3 months.

At present there does not appear to be any reason to administer thiamin intraspinally. In our experience thiamin is rapidly transmitted from the blood into the cerebrospinal fluid, where it may be found in increasing amounts 1 hour after intravenous injection.

The clinical response to vitamin B1 therapy is often dramatic. In the acute cases beginning improvement may be noticed within a few hours; the mild cases are often relieved within 24 to 48 hours. In the chronic case there is often great relief, particularly from pain, within a few days, though other signs may remain for a long period. Occasionally, in chronic cases, some residual changes remain indefinitely. It is generally recognized that the maintenance requirement and the curative dose of vitamin B1 vary from one individual to another and may vary in the same individual from time to time. These variabilities necessitate close medical supervision at all times.

\section{Pellagra}

\section{Nicotinic Acid Deficiency}

Mental changes as a part of the pellagra syndrome have been recognized by many physicians, and in areas where the disease is endemic these symptoms are so common and so striking that they have become associated with pellagra even by the lay observer. Various abnormal psychic states have been described in medical literature on pellagra, and some writers have thought that one or another psychosis was typical of this disease. Subclinical pellagrins are noted for the multiplicity of their complaints, among which are many that are usually classified as neurasthenic. Practically every patient who finally had lesions characteristic of pellagra had been diagnosed previously as neurasthenia. The conduct of the pellagrin may be normal ; but he feels incapable of mental or physical effort, even though he may be ambulatory.

The noticeable and more serious mental signs of pellagra manifest themselves in various types of psychoses. The most common is perhaps that in which loss of memory, disorientation, confusion, and confabulation are predominant. There are also types in which excitement, mania, depression, and delirium may occur. In our experience a paranoid condition is common in pellagrins. These patients, acting on their paranoid delusions, are more active than are other pellagrins with psychoses.

Elvehjem, Madden, Strong, and Woolley (1937) reported that nicotinic acid cured blacktongue in dogs, a canine disease which is believed to be analogous to pellagra in human beings. Since all food substances which have been found 
to be curative and preventive for canine blacktongue have likewise proved beneficial in the treatment and prevention of human pellagra, investigators were encouraged to determine the effect of nicotinic acid on this disease. Spies, Cooper, and Blankenhorn (1938) showed that pellagrins responded dramatically to the administration of this drug. Beneficial results had also been observed independently by other investigators in the field. Spies, Sasaki, and Gross (1938) and Spies, Bean, and Stone (1938) mentioned briefly that the early and late mental symptoms of pellagrins in relapse and of sub-clinical pellagrins were relieved by nicotinic acid. In a larger series of cases it was possible to institute control measures in order to study more specifically the mental symptoms of pellagrins in relapse and their response to nicotinic acid. Related studies on subclinical and mild cases have also been made.

\section{Acute Psychoses}

We have been able to relieve quickly the acute psychoses of 70 pellagrins with the use of nicotinic acid (Spies, Aring, Gelperin, Bean, 1938). The psychosis had been present from 1 to 83 days in these cases, and in 10 of these patients the psychosis had been induced in the hospital. Five hundred to $1000 \mathrm{mg}$. of nicotinic acid was administered daily to these patients, in $50 \mathrm{mg}$. doses.

The relief of the numerous and varied abnormal mental signs in this group of pellagrins was dramatic in its suddenness, and was maintained as long as nicotinic acid or a well-balanced diet supplemented with nicotinic acid was administered. There was no instance in these 70 psychotic pellagrins in which we were unable to relieve the psychosis. Further experience has upheld the efficacy of nicotinic acid in the treatment of this type of patient. The amide of nicotinic acid (sodium nicotinate) acts similarly to nicotinic acid in these cases.

\section{Chronic Psychoses}

We were unable to relieve completely the chronic psychoses accompanying pellagra in 13 cases. Large doses (500 to $1000 \mathrm{mg}$. daily) of nicotinic acid were administered for periods varying from $1 \frac{1}{2}$ to $2 \frac{1}{2}$ months to these pellagrins suffering from chronic psychoses. These patients became more alert, cooperative, and tidy, and apathy and restlessness disappeared. Most of these patients changed from bedridden to ambulatory patients. Similar beneficial results have been obtained recently following the administration of similar doses of either the monocarboxylic acid of pyrazine, or quinalinilic acid.

Several reports (Evans, 1939 ; Boga-t, 1938 ; Frontali and Ferrari, 1938) have appeared on the beneficial effect of nicotinic acid in the psychoses of pellagra. There has been no report of the complete relief of a chronic pellagrous psychosis of many years' duration. In only two cases, who had been in mental hospitals for 9 and 8 months respectively, were we able to effect anything approaching complete cure of the chronic psychoses of pellagra. These cases are too few on which to base a final opinion. 
"Neurasthenia"

Nicotinic acid was also administered to a group of 225 mild pellagrins, with complete relief of their rather typical complaints, which have usually been considered to be those of neurasthenia (Spies, Aring, Gelperin, and Bean, 1938). The more common symptoms in this multiplicity of complaints are fatigue, insomnia, anorexia, vertigo, burning sensations in various parts of the body, numbness, palpitation, nervousness, a feeling of unrest and anxiety, headache, forgetfulness, apprehension, and distractibility. On nicotinic acid therapy, the ambulatory pellagrin also regains energy and appetite. This relief occurs within 1-12 days following the administration of from three to six doses, $100 \mathrm{mg}$. each, of nicotinic acid per day.

\section{Stupor}

It should be emphasized that many people develop the mental signs of pellagra, at least to a mild degree, long before they develop other manifestations of the disease. We have treated with nicotinic acid 60 cases, who had mental symptoms ranging from psychoses to stupor, without any clinical evidence of pellagra other than malnutrition and a positive test for porphyrins or related substances in the urine. This test (Beckh, Ellinger, and Spies, 1937) is suggestive of a nutritional deficiency, but not diagnostic of pellagra. These patients were promptly relieved by nicotinic acid. Eleven other cases with stupor or psychoses with a negative urinalysis responded to nicotinic acid. Our experience and that of Sydenstricker, Schmidt, Fulton, New, and Geeslin (1938) suggests that it is advisable to use nicotinic acid as a therapeutic test in such individuals. Five hundred to $1000 \mathrm{mg}$. given in 10 equal doses during each 24 hours for a period of 2 weeks is inexpensive and may result in a miraculous cure.

\section{Deafness}

Selfridge (1939) reported improvement in the hearing of patients with high-tone deafness with the use of nicotinic acid. We have observed that pellagrins are better able to hear after the administration of large amounts of nicotinic acid, probably due to an improvement in their mental state and a diminution in their tinnitus and dizziness.

\section{Chemistry of Nicotinic Acid}

Nicotinic acid, as well as thiamin hydrochloride and riboflavin, are components of important intracellular enzymes.

Nicotinic acid in its amide form is an important constituent of cozymase (von Euler, Hebers, and Schlenck, 1935) and of coferment (Warburg and Christian, 1936). It has been shown (Vilter, Vilter, and Spies, 1939) that in severe pellagra in relapse the blood level of codehydrogenases I and II (cozymase and coferment) is below normal and that these coenzymes may be returned to their normal levels in the blood by the administration of nicotinic acid or its amide. It would seem that persons with pellagra have less of these fundamental enzymes in their tissues than do normal persons. Evidently nicotinic 
acid is changed into nicotinic acid amide, and nicotinic acid amide is built into the molecule of cozymase and coferment. This might account for the delay which occurs between the administration of nicotinic acid and the elevation of these substances in the blood.

\section{Prevention and Treatment of Nicotinic Acid Deficiency}

The dietary advice noted under the prevention and treatment of thiamin deficiency is as applicable to the pellagrin as to those suffering from vitamin B1 deficiency.

The optimum dosage of nicotinic acid, like that of thiamin, has not been determined. Neither has the minimal dose of nicotinic acid been determined but it seems to vary from case to case. It is thought that patients with severe mental symptoms should receive a total of at least $500 \mathrm{mg}$. per day. This is best administered by mouth in 10 equal doses, although it may be administered parenterally. In the extremely severe cases convalescence may be shortened by the administration of $1,000 \mathrm{mg}$. daily. We have administered as much as $2,000 \mathrm{mg}$. daily in divided doses without untoward results. There may be some flushing and feeling of warmth in the skin incident to large doses of nicotinic acid.

The dosage of nicotinic acid amide and sodium nicotinate is similar to that of nicotinic acid.

Nicotinic acid may be administered intravenously in dilute solutions. The nicotinic acid should be dissolved in sterile physiological solution of sodium chloride. Nicotinic acid may be given intramuscularly with slight discomfort. A solution containing $5 \mathrm{mg}$. of nicotinic acid per c.c. of normal saline should be used. These solutions may be sterilized by boiling or autoclaving, as nicotinic acid is heat stable. The total daily dose of nicotinic acid when given parenterally varies from 40 to $80 \mathrm{mg}$. This total dosage should be given in divided doses of 10-15 mg. each.

\section{Riboflavin (Vitamin B2) Deficiency}

The use of riboflavin in the human (Sebrell and Butler, 1938 ; Vilter, Vilter, and Spies, 1939) has been in conditions other than those associated with neurological disease. We have seen peripheral neuritis become worse in persons with the healing lesions of riboflavin deficiency (roughening of the skin around the mouth and across the tip of the nose) during the administration of riboflavin. The amounts of riboflavin given to cure these lesions do not affect the peripheral neuritis; also peripheral neuritis develops in persons getting large amounts of riboflavin but eating a diet deficient in vitamin B.

\section{Vitamin B6 Deficiency}

This substance, recently synthesized in small amounts, has been used in a study (Spies, Bean, and Ashe, 1939) of four cases of nutritional deficiency. In instances of multiple deficiency the addition of thiamin chloride, nicotinic acid, and riboflavin to the inadequate diet produced great relief from beriberi, 
pellagra, and riboflavin deficiency. If the diet is unchanged symptoms may remain or develop which are not corrected by these substances. The most obvious symptoms are irritability, insomnia, nervousness, vomiting, weakness and difficulty in walking. The gait may resemble that seen in acute cerebellar disorders ; it is wide-based and reeling. Cachexia may supervene.

Spies, Bean, and Ashe (1939) administered $50 \mathrm{mg}$. of synthetic vitamin B6 in sterile physiologic solution of sodium chloride intravenously to four of these patients, with abrupt improvement in all of them. Within 24 hours the symptoms, with the exception of the cachexia, disappeared. One of these individuals who could stagger only a few steps walked two miles within 24 hours after the injection of $50 \mathrm{mg}$. of vitamin B6. All patients noted conspicuous gain of strength and a feeling of well-being after a single injection.

Observations are being made on the possible relationship of this substance to the nervous diseases due to nutritional deficiency.

\section{Multiple Vitamin Deficiency}

We have discussed the deficiencies of portions of the vitamin B complex and the nervous manifestations therefrom in terms of distinct entities. As one's experience with these diseases increases the realization becomes stronger that the majority of patients suffering from nutritional deficiency are affected by a lack of more than one substance. This point of view has already been suggested by the clinical work that has proceeded with vitamin B6 and which has been described in a preceding paragraph.

In the Nutrition Clinic at the Hillman Hospital in Birmingham, Alabama, close watch has been kept on the pellagrins for symptoms of beriberi and riboflavin deficiency. It has been found that practically all pellagrins have, or have had, symptoms of peripheral neuritis. If these symptoms are absent, abnormality of the nerves can be demonstrated in active pellagrins with a chronaximeter (Lewy, Aring, and Spies, 1939). The neuritis in ambulatory pellagrins is, as a rule, not severe and is usually limited to the lower extremities. It can be made to regress and reappear, depending upon the administration or the witholding of thiamin chloride.

Symptoms of riboflavin deficiency in pellagrins, while not as common as those of beriberi, occur often enough to warrant its inclusion in the conception of multiple deficiency.

It is to be expected that nutritional disorders should be associated one with the other. In foodstuffs the water-soluble vitamins are often associated, and a diet deficient in one substance is almost certain to be deficient in others. Inadequate and unbalanced diets eaten by the majority of patients predispose them simultaneously to a number of nutritional disorders, and the diagnosis of one clinical deficiency syndrome necessitates a thorough search for others.

The multiple nutritional deficiencies have been studied (Spies, Vilter and Ashe, 1939) chiefly by administering a pure vitamin and observing the relief of certain of the symptoms, diagnostic of the syndrome for which the agent is specific, but no improvement of the lesions of associated syndromes, so long as the diet of the patient remains constant. 


\section{Pathology of Avitaminosis}

This portion of the subject has been covered recently by Greenfield (1938) in this journal.

In our studies of the biopsies of peripheral nerve in 12 cases of deficiency disease with neuritic symptoms, in several instances both before and months after treatment, there have occurred loss and degeneration of myelin sheaths in the terminal portions of the anterior tibial nerve. We have not seen this degeneration in its active phase, but only after the destruction and removal of the products of degeneration had occurred. The axones in these peripheral nerves on the whole have been normal. In those cases in which the peripheral nerve was examined as long as 11 months after treatment was instituted, the myelin sheath loss was as severe as it was before treatment was begun. This relationship of vitamin B1 deficiency to degeneration of the peripheral nerves is well known. Bertrand, Liber, and Randoin (1934) found that the katabolism of myelin in a degenerating nerve ceased in pigeons deficient in vitamin B1. Associated with this phenomenon was inactivity of the sheath cells of Schwann. After treatment with vitamin B1 the Schwann cells became active and phagocytosis of the degeneration products of myelin occurred. Vitamin B1 appears to be a requisite in the repair of the nerve fibre.

In recent years, however, there has been a strong tendency to consider many of the pathological changes of experimental beriberi the result of starvation rather than the result of a vitamin B1 deficiency. Particularly significant in support of this idea is the general opinion among investigators that one of the earliest results of a deprivation of vitamin B1 is failure of appetite. Chamberlain, Bloombergh, and Kilbourne (1911) demonstrated that fowls dying from starvation had degeneration of the peripheral nerves which could not be distinguished readily from the degeneration found in polyneuritis gallinarum induced by an exclusive rice diet. Davison and Stone (1937) believe that the pathological changes in the nervous system in animals suffering from inanition are essentially the same as those in animals deprived of vitamin B1 or B2. Vedder and Chinn (1938) compared the brachial and sciatic nerves of rats subjected to complete starvation, rats completely starved but given $3 \mathrm{mg}$. of crystalline vitamin B1 orally each day, and rats given a diet deficient in vitamin B1. Myelin degeneration was seen in the peripheral nerves of all rats of all groups. No appreciable difference in the extent of this degeneration was noted in the three groups. Sebrell and Elvove (1931) have shown that an inadequate amount of vitamin B1 added to the vitamin B1 free diet of rats makes the neuritis more severe than if no vitamin B1 is added. It is essential that further studies on the physiological action of vitamin B1 be made before we can determine how definite a role inanition plays in the development of polyneuritis in vitamin B1 deficient animals.

Human beings, however, often develop the lesions while maintaining normal body weight. From studies on human beings it is thought by the authors that diets deficient in other essential factors increase the requirement for vitamin B1 and that this substance is not stored in the body to the extent that we previously 
thought. It would seem, therefore, that these factors play a significant role in the development of clinical beriberi in human beings.

Experimental polyneuritis in fowls usually is in many ways comparable to dry beriberi in man. In the experimental animal, as in the human being with vitamin B1 deficiency, the greatest degeneration is in the peripheral nerves, especially the distal portions, the sciatic nerves being particularly affected. Vedder and Clark (1912) showed that in experimental neuritis practically every fibre of the nerve has some evidence of degeneration, though the extent varies greatly in the fibres of the same nerve. The myelin is degenerated and there is swelling of the nerve-sheath in 10 to 15 per cent. of the fibres. Vedder and Clark described the earliest stage of myelin degeneration as slight swelling of the medullary sheath and a tendency toward segmentation at the circumference. There are generalized changes affecting the peripheral nerves, the ventral and dorsal nerve roots, all tracts of the spinal cord, the medulla, pons, midbrain, and internal capsule. There is also degeneration of the nerve cells. These changes in the nervous system appear similar to those described in human beriberi. It is thought, however, that in order to produce lesions similar to or identical with those appearing in human beings some vitamin B1 must be fed to the experimental animal in order to prevent early death.

The nervous lesions of pellagra are not as definite as those associated with lack of vitamin B1. Goldberger, Wheeler, Lillie, and Rogers (1928) produced in dogs a condition associated with black tongue which they thought resembled human pellagra, but they did not reproduce the nervous lesions of the human disease. Only slight degeneration was found in the peripheral nerves and dorsal columns in those dogs that survived longest. Stern and Findlay (1929) produced in rats a similar condition associated with lesions of the epidermis and some degree of paralysis, but without definite changes in the peripheral nerves or tract lesions in the spinal cord. Zimmermann (1937) used a diet from which he believed vitamin B2 was excluded. In the more chronic disease which he produced the degeneration was very marked, both in the peripheral nerves and dorsal columns. The similarity of these changes to those seen in cases of pellagra is evidence of the relation of this disease to lack of vitamin B2, although there are differences between the human and the experimental disease. Zimmermann stated that the anterior horn cells and Betz cells in his dogs were normal, whereas in the human disease the latter cells suffer, along with many other cortical neurones, from the chromolytic changes which Adolf Meyer (1901) called "central neuritis." On the other hand, Stern and Findlay found changes in the neurones, but none in the tracts of the cord or peripheral nerves in rats deprived of vitamin B2. Bessey, Boyle, and Wolbach (1937) have confirmed this work with rats. Obviously rats respond differently.

In the human disease the long tracts of the cord may or may not be affected. This is possibly a factor which influences the severity and duration of the pellagra. Usually the dorsal columns are more affected than the anterio-lateral columns, but in a case reported by Langworthy (1931) the reverse was the case. In Greenfield's (1938) cases the degeneration appeared to differ from that of subacute combined degeneration in that there was very little evidence of 
vacuolation in the early stages. It seemed to be a true Wallerian degeneration, and in the dorsal columns was related to degeneration of the entering spinal roots. In some of Pentschew's (1929) cases in which the duration of the illness was longer, asymmetrical areas of sclerosis were found on the margins of the antero-lateral columns.

\section{Persistent Problems}

If it has not been indicated already, many of the concepts are unsettled in the field of nutritional deficiency. The number of problems that remain to be solved furnishes an index of our comparative ignorance of the vitamin deficiencies that affect the nervous system. The more pressing problems awaiting the worker in this field will be described briefly.

The relationship to nervous disease, of deficiency of vitamins besides those of the B complex that have been synthesized, has not been explored in man. This is no doubt due to the inability to obtain sufficient quantities of the pure vitamin. The work of Mellanby (1934) with vitamin A and of Einarson and Ringsted (1938) with vitamin $\mathrm{E}$ in animals is provocative.

The definite relief of pain (neuralgias, tabes), in certain instances, with the use of vitamin B1 needs elucidation. Vitamin B1 does not appear to be an analgesic (except in patients with nutritional neuritis). Neuralgia and tabes dorsalis do not appear to be vitamin deficiencies. In the experience of many workers the neuralgias are not benefited by the use of vitamin B1. It is possible that the concept of nutritional deficiency may have to be expanded in some instances to include many diseases now thought to be outside of its scope.

Why is it wellnigh impossible to produce neuritis in a normal man not previously deficient in vitamins by the use of diets nearly totally deficient in vitamin B1 ? It is well known that vitamin B1 is not stored to any extent by the body.

In consideration of the frequency with which acute psychotic episodes occur in pellagra, why are the chronic psychoses so rare in pellagrins who have had the disease for years? The paucity of cases of psychoses due to pellagra in the custodial mental hospitals in the State of Alabama is striking.

There is disagreement about the neuropathology of the vitamin deficiencies in man. Possibly the conception that the great majority of the nutritional deficiencies are deficiencies of many vitamins may aid in the interpretation of the varying pathological findings. A report of the neuropathological lesions found in the cases that we have studied will be published at a later date.

\section{Summary}

Vitamin B1 is more appropriately termed the " anti-neuritic vitamin" than would be any other single nutritional element. Careful and controlled work has demonstrated the necessity for vitamin B1 in the repair of nerves damaged by a lack of this vitamin in the diet. The rapid relief of the discomfort of neuritis and therefore improved motor function in man suffering from nutritional 
neuritis is a common observation. This improvement is most likely humoral in nature. Identical histological changes have been demonstrated in man in peripheral nerve twigs before and at varying intervals after symptomatic response to treatment.

It is unlikely that lack of vitamin B1 is the sole cause of nutritional neuritis in man, as one can reach a certain stage with thiamin therapy, beyond which improvement does not progress with continued vitamin Bl administration. This same type of improvement may be obtained in cases of neuritis now thought to be due to causes other than nutritional deficiency (brachial neuritis, emetine neuritis).

Our experience leads us to believe that if vitamin B1 is to be effective in any very serious nervous disease it must be given in large doses. We recommend the administration of at least 50-100 mg. each day, given parenterally in several doses of $10-20 \mathrm{mg}$. each. It is also our belief that if no response is obtained within a week, further vitamin B1 therapy will be unavailing.

Nicotinic acid has been proved to be a potent substance in the relief of the somatic complaints and of the acute psychoses associated with pellagra. Cases with chronic psychoses and pellagra are almost invariably benefited by this portion of the B complex and, in rare instances, are cured.

It has been suggested that thiamin and nicotinic acid which are perhaps essential to all cell life act through fundamental oxidative-reductive mechanisms of the body, the former through cocarboxylase, the latter through coenzyme or coferment. Since normal intracellular metabolism depends in large part upon the close interrelation of many enzymes, we may postulate that the pathological physiology of the deficiency states will be better understood when we have final information as to the nature and function of these enzymes.

Perhaps nowhere in the field of medicine is such prompt and lasting relief of symptoms obtained as that which follows administration of adequate amounts of these materials. While they cut down the time required for convalescence it must always be kept in mind that the administration of the vitamins does not replace the well-balanced diet.

\section{REFERENCES}

Alexander, L., Myerson, A., and Pijoan, M. (1938). Beri-beri and Scurvy. Trans. Amerneurol. Ass.

Beckh, W., Ellinger, P., and Spies, T. D. (1937). Ouart. J. Med., 6, 305.

Bertrand, I., Liber, A. F., and Randoin, L. (1934). Arch. Anat. micr., 30, 297.

Bessey, O. A., Boyle, P. E., and Wolbach, S. B. (1937). In Wolbach, S. B., J. Amer. med. Ass., 108, 7.

Bogart, C. N. (1938). Ibid., 111, 613.

Brown, M. R. (1938). Med. Clin. N. Amer., 22, 627.

Chamberlain, W. P., Bloombergh, H. D., and Kilbourne, E. D. (1911). Philipp. J. Sci., 6, 177.

Cline, T. K. (1938). In Williams and Spies, Vitamin B1, MacMillan, N.Y.

Cobb S., and Coggeshall, H. C. (1934). J. Amer. med. Ass., 103, 1,608.

Council of Pharmacy and Chemistry (1936). "The Status of Certain Questions concerning Vitamins," Ibid., 106, 1,732.

Davison, C., and Stone, L. (1937). Arch. Path. Lab. Med., 23, 207.

Durand, J. I., Spickard, V. W., and Burgess, E. (1939). J. Pediat., 14, 74.

Einarson, L., and Ringsted, A. (1938). Effect of Chronic Vitamin E Deficiency on the Nervous System and the Skeletal Musculature in Adult Rats. Oxford Univ. Press, London, pp. 163. 
Elvehjem, C. A., Madden, R. J.. Strong, F. M., and Woolley, D. W. (1937). J. Amer. chem. Soc., 59, 1,767.

Evans, V. L. (1939). J. Amer. med. Ass., 112, 1,249.

Frontali, G., and Ferrari, G. (1938). Minerva med. Rome, 2, 133.

Glick, D. and Antopol, W. (1939). J. Pharmacol., 65, 389.

Goldberger, J., Wheeler, G. A., Lillie R. D., and Rogers, L. M. (1928). U.S.A. Pub. Hlth. Rep., 43, 687 and 1,385.

Greenfield, J. G. (1938). J. Neurol. Psychiat., 1, 306.

Greenfield, J. G., and Carmichael, E. A. (1935). Brain, 58, 483.

Kepler, E. J. (1925). J. Amer. med. Ass. 85, 409.

Langworthy, O. R. (1931). Brain, 54, 291.

Lewy, F. H., Aring, C. D., and Spies, T. D. (1939). Unpublished observations.

Lewy, F. H., Himwich, H. E., Frostig, J. P., and Spies, T. (1939), Science, 90, 141.

Mellanby, E. (1934). Nutrition and Disease. Oliver and Boyd, London and Edinburgh.

Metildi, P. F. (1939). Amer. J. Syph., 23, 1.

Meyer, A. (1901). Brain., 24, 47.

Minz, B. (1938). Presse méd., 46, 1,406.

Pentschew, A. (1929). Z. ges. Neurol. Psychiat., 118, 17.

Schiro, H., Spies, T. D., and Aring, C. D. (1939). Unpublished observations.

Sciclounoff, F., and Broccard, R. (1936). Schweiz. med. Wschr., 66, 985.

Sebrell, W. H. and Butler, R. E. (1938). Publ. Hlth. Rep., Wash., 53, 2,282.

Sebrell, W. H., and Elvove, E. (1931). U.S.A. Publ Hlth. Rep., 46, 917.

Selfridge, G. (1939). Ann. Otol., etc. St. Louis, 48, 39.

Sindoni, A. (1936). Amer. J. Digest. Dis. Nutrit., 3, 759.

Spies, T. D. (1939). Sth. med. J., 32, 618.

Spies, T. D., and Aring, D. C. (1938). J. Amer. med. Ass., 110, $1,081$.

Spies T. D., Aring, D. C., Gelperin, J., and Bean, W. B. (1938). Amer. J. med. Sci., 196, 461.

Spies, T. D., Bean, W. B., and Ashe, W. F. (1939). J. Amer. med. Ass., 112, 2,414-15.

Spies, T. D., Bean W. B., and Stone, R. E., (1938). Ibid., 111, 584.

Spies, T. D., Cooper, C., and Blankenhorn, M. A. (1938). Ibid., 110, 622.

Spies, T. D., Saski, Y., and Gross, E. S. (1938). Sth. med. J., 31, 483.

Spies, T. D., Vilter, R. W., and Ashe, W. F. (1939). J. Amer. med. Ass., in press.

Stern, R. O., and Findlay, G. M. (1929). J. Path. Bact., 32, 63.

Sydenstricker, V. P., Schmidt, H. L., Fulton, M. C., New, J. S., and Geeslin, L. (1938). Sth. med. J., 31, 1,155.

Urmy, T. V., Ragle, B. H., Allen, A. W., and Jones, C. M. (1934). New Eng. J. Med., 210, 251.

Vedder, E. B., and Chinn, A. B. (1938). In Williams and Spies, Vitamin B1, MacMillan, N. Y., p. 58 .

Vedder, E. B., and Clark, E. (1912). Philipp. J. Sci., 7, 423.

Vilter, R., Vilter, S. P., and Spies, T. D. (1939). J. Amer. med. Ass., 112, 420.

von Euler, H., Hebers, H., and Schlenck, F. (1935). Z. physiol. Chem., 237, 1.

Vorhaus, M. G., Williams, R. R., and Waterman, R. E. (1935). J. Amer. med. Ass., 105, 1,580 .

Warburg, O., and Christian, W. (1936). Biochem. Z., 285, 156.

Williams, R. R., and Cline, J. K. (1936). J. Amer. chem. Soc., 58, 1,054.

Williams R. R., and Spies, T. D. (1938). Vitamin B1, MacMillan, N.Y.

Williams, R. R., Waterman, R. E., and Keresztesy, J. C. (1934). J. Amer. chem. Soc., 56, 1,187 .

Zimmerman, H. M., (1937). Schweiz. Arch. Neurol. Psychiat., 39, 195. 